Accepted for publication, The Astronomical Journal. 2008 November 28.

Preprint typeset using $\mathrm{LAT}_{\mathrm{E}} \mathrm{X}$ style emulateapj v. 08/22/09

\title{
TIME-RESOLVED NEAR-INFRARED PHOTOMETRY OF EXTREME KUIPER BELT OBJECT HAUMEA
}

\author{
PEDRo LACERDA \\ Institute for Astronomy, University of Hawaii, 2680 Woodlawn Drive, Honolulu, HI 96822 \\ Accepted for publication, The Astronomical Journal. 2008 November 28.
}

\begin{abstract}
We present time-resolved near-infrared $(J$ and $H$ ) photometry of the extreme Kuiper belt object (136108) Haumea (formerly $2003 \mathrm{EL}_{61}$ ) taken to further investigate rotational variability of this object. The new data show that the near-infrared peak-to-peak photometric range is similar to the value at visible wavelengths, $\Delta m_{R}=0.30 \pm 0.02$ mag. Detailed analysis of the new and previous data reveals subtle visible/near-infrared color variations across the surface of Haumea. The color variations are spatially correlated with a previously identified surface region, redder in $B-R$ and darker than the mean surface. Our photometry indicates that the $J-H$ colors of Haumea $(J-H=-0.057 \pm 0.016$ mag) and its brightest satellite Hi'iaka $(J-H=-0.399 \pm 0.034 \mathrm{mag})$ are significantly $(>9 \sigma)$ different. The satellite Hi'iaka is unusually blue in $J-H$, consistent with strong $1.5 \mu \mathrm{m}$ water-ice absorption. The phase coefficient of Haumea in the $J$-band is found to increase monotonically with wavelength in the range $0.4<\lambda<1.3$. We compare our findings with other Solar system objects and discuss implications regarding the surface of Haumea.
\end{abstract}

Subject headings: Kuiper belt — methods: data analysis - minor planets, asteroids — solar system: general - techniques: photometric

\section{INTRODUCTION}

Kuiper belt objects (KBOs) orbit the sun in the transNeptunian region of the Solar system. Mainly due to their large heliocentric distances and resulting low temperatures, KBOs are amongst the least processed relics of the planetary accretion disk and thus carry invaluable information about the physics and chemistry of planet formation. Moreover, as a surviving product of the debris disk of the Sun, the Kuiper belt is a nearby analog to debris disks around other stars and may provide useful insights into the study of the latter.

The known KBO population - which currently amounts to over a thousand objects - provides several clues to the origin and evolution not only of the small bodies but also of the planets. One example is the outward migration of planet Neptune, inferred from the need to explain the resonant structure of the KBO population, namely the 3:2 resonant KBOs of which (134340) Pluto is a member (Malhotra 1995). Extreme, physically unusual objects are a profitable source of interesting science as they often challenge existing paradigms. One such unusual object in the Kuiper belt is (136108) Haumea, formerly known as $2003 \mathrm{EL}_{61}$.

Haumea is remarkable in many ways. With approximate triaxial semi-axes $1000 \times 800 \times 500 \mathrm{~km}$, it is one of the largest known KBOs. Its elongated shape is a consequence of the very rapid $3.9 \mathrm{~h}$ period rotation, and those two properties combined can be used to infer Haumea's bulk density $\left(\rho \sim 2500 \mathrm{~kg} \mathrm{~m}^{-3}\right)$, assuming that the object's shape has relaxed to hydrostatic equilibrium (Rabinowitz et al. 2006; Lacerda \& Jewitt 2007). Haumea's rapid rotation and the spectral and orbital similarity between this object and a number of smaller KBOs, have led Brown et al. (2007) to suggest that an ancient shattering collision ( $>1$ Gyr ago;
Ragozzine \& Brown 2007) could explain both. Haumea is one of the bluest known KBOs, with $B-R=0.97 \pm 0.03$ mag (Rabinowitz et al. 2006; Lacerda et al. 2008), and it has an optical and infrared spectrum consistent with a surface coated in almost pure water-ice (Tegler et al. 2007; Trujillo et al. 2007). This stands in contrast with other large KBOs such as Pluto, Eris, and $2005 \mathrm{FY}_{9}$, which have methane rich surfaces (Cruikshank et al. 1976; Brown et al. 2005; Licandro et al. 2006). Two satellites have been detected in orbit around Haumea. The innermost, Namaka, has an orbital period of $P_{\text {orb }} \sim$ 34 days, an apparent orbital semimajor axis $a \sim 1^{\prime \prime}$, and a fractional optical brightness of $f \sim 1.5 \%$ with respect to Haumea. The outermost, Hi'iaka has $P_{\text {orb }} \sim 41$ days, $a \sim 1.2^{\prime \prime}$, and $f \sim 6 \%$ (Brown et al. 2006).

Time-resolved optical photometry of Haumea has revealed evidence for a localized surface feature both redder and darker than the surrounding material (Lacerda et al. 2008). Although the existing data are unable to break the degeneracy between the physical size and the color or albedo of this dark, red spot (hereafter, DRS), the evidence points to it taking a large $(>20 \%)$ fraction of the instantaneous cross section. The composition of the DRS remains unknown but its albedo and $B-R$ color are consistent with the surfaces of Eris, $2005 \mathrm{FY}_{9}$, and Pluto's and Iapetus' brighter regions. These observations motivated us to search for rotational modulation of the water ice band strength that might be associated with the optically detected DRS.

In this paper we provide further constraints on the surface properties of Haumea. We present time-resolved near-infrared ( $J$ and $H$ ) data and search for visible/nearinfrared color variability. We also constrain the $J$-band phase function of Haumea and compare it to its optical counterparts. Finally, we measure the $J-H$ color of Hi'iaka, the brightest satellite of Haumea. 
TABLE 1

Journal of ObSeRvations of Haumea.

\begin{tabular}{ll}
\hline UT Date & $2008 \mathrm{Apr} 14$ \\
Heliocentric Distance, $R$ & $51.116 \mathrm{AU}$ \\
Geocentric Distance, $\Delta$ & $50.240 \mathrm{AU}$ \\
Phase angle, $\alpha$ & $0.55^{\circ}$ \\
Weather & Photometric \\
Telescope & $8.2 \mathrm{~m}$ Subaru \\
Instrument & MOIRCS \\
Pixel scale & $0.117^{\prime \prime} /$ pixel \\
Seeing & $0.5^{\prime \prime}-0.8^{\prime \prime}$ \\
Filters (Exp. Time) & $J(30 \mathrm{~s}), H(20 \mathrm{~s})$ \\
\hline
\end{tabular}

\section{OBSERVATIONS}

Near-infrared observations were taken using the 8.2$\mathrm{m}$ diameter Subaru telescope atop Mauna Kea, Hawaii. We used the Multi-Object Infrared Camera and Spectrograph (MOIRCS; Tokoku et al. 2003) which is mounted at the $\mathrm{f} / 12.2$ Cassegrain focus. MOIRCS accomodates two $2048 \times 2048$ pixel $\mathrm{HgCdTe}$ (HAWAII-2) arrays, with each pixel projecting onto a square $0.117^{\prime \prime}$ on a side in the sky. Observations were obtained through broadband $J\left(\lambda_{\mathrm{c}}=1.26 \mu \mathrm{m}, \Delta \lambda=0.17 \mu \mathrm{m}\right)$ and $H\left(\lambda_{\mathrm{c}}=1.64 \mu \mathrm{m}\right.$, $\Delta \lambda=0.28 \mu \mathrm{m})$ filters. The data were instrumentally calibrated using dark frames and dome flat-field images obtained immediately before and after the night of observation. Because of technical difficulties with detector 1 , we used detector 2 for all our science and calibration frames. Science images were obtained in sets of two dithered positions $15^{\prime \prime}$ apart, which were later mutually subtracted to remove the infrared background flux.

The night of 2008 April 15 UT was photometric, allowing us to absolutely calibrate the data using observations of standard star FS33 from the UKIRT Faint Standards catalog (Hawarden et al. 2001). The Haumea flux through each filter was measured using circular aperture photometry relative to a field star, while a second field star was used to verify the constancy of the first. The dispersion in the star-to-star relative photometry indicates a mean $1 \sigma$ uncertainty of \pm 0.015 magnitude in $J$ and \pm 0.023 magnitude in $H$. The field star was calibrated to the standard star FS33 at airmass 1.02, just short of the telescope's Alt-Az elevation limit. From scatter in the standard star photometry we estimate a systematic uncertainty in the absolute calibration of 0.04 magnitudes in $J$ and 0.02 magnitudes in $H$. A brief journal of observations can be found in Table 1]. The final calibrated broadband photometric measurements are listed in Tables 2 and 3 .

We generally obtained two consecutive sets of two dithered images in each filter before switching filters (i.e. $J J-J J-H H-H H-\ldots)$. This results in sets of four data points all within 3 to 4 minutes of each other. To reduce the scatter in the lightcurves we binned each of these sets of consecutive measurements into a single data point, with each binned point obtained by averaging the times and magnitudes of the set. The error bar on each binned point includes the error on the mean magnitude and the average uncertainty of the unbinned measurements, added in quadrature. The binned measurements are listed in Tables 4 and 5 .

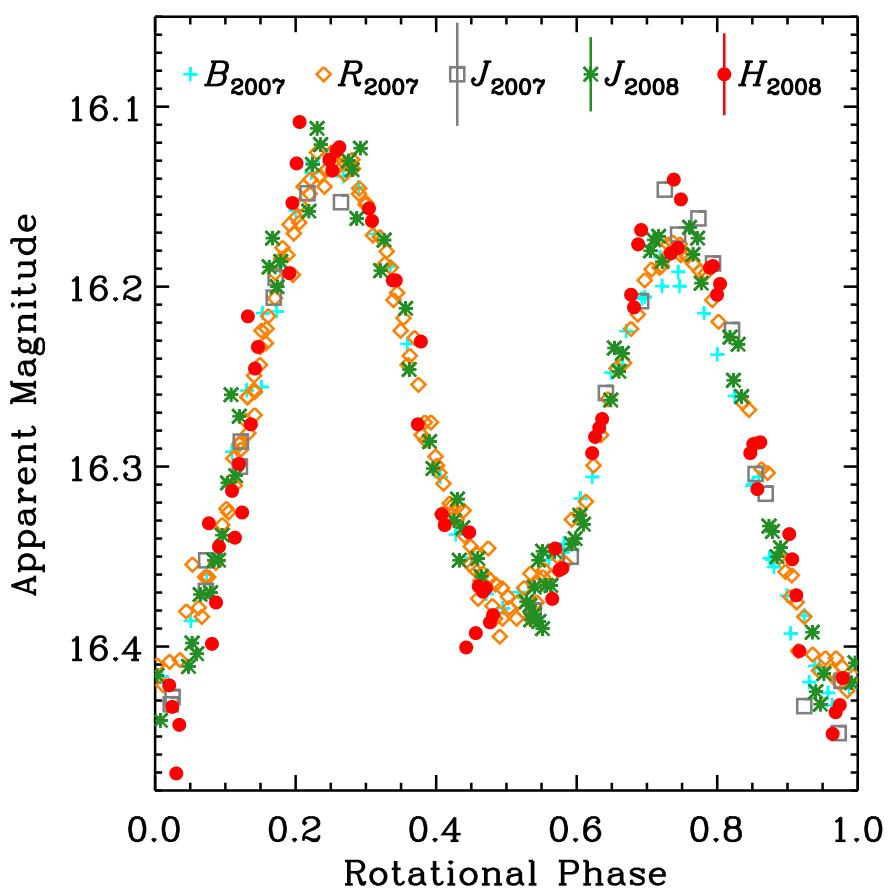

FIG. 1. - Four-color lightcurve of Haumea. Symbols labelled 2008 correspond to the new $J$ and $H$ data presented here. Measurements from 2007 (Lacerda et al. 2008) are marked for direct comparison. The vertical axis corresponds to the $J_{2008}$ apparent magnitude. Data in other bands have been shifted using the mean colors (see text).

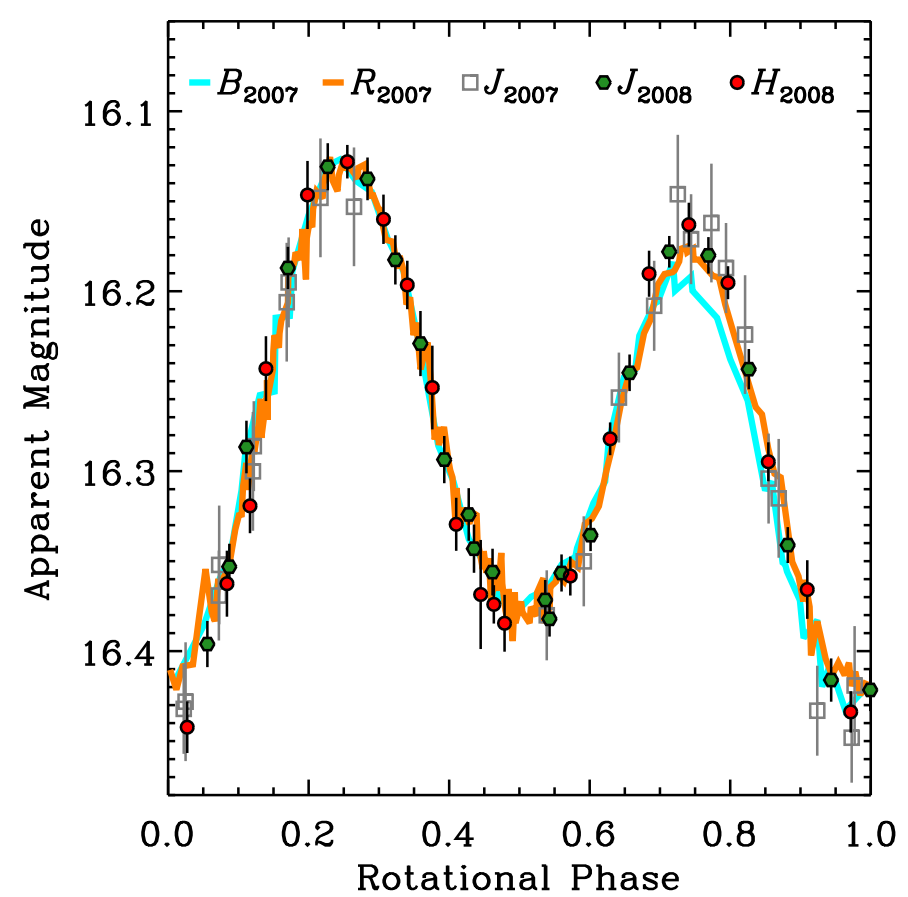

FIG. 2.- Binned near-infrared lightcurve of Haumea. Here, sets of consecutive data points in Fig. 1 have been binned to reduce scatter. Green hexagons ( $J$-band) and red circles $(H$-band) mark the mean rotational phase and magnitude of consecutive measurements (mostly sets of four). Measurements from 2007 (Lacerda et al. 2008) are marked for direct comparison: the green squares are $J$-band measurements, while the densely sampled $B$ - and $R$-band data are plotted as thick cyan and orange lines. The vertical axis corresponds to the $J_{2008}$ apparent magnitude. Data in other bands have been shifted using the mean colors (see text). 
TABLE 2

$J$-band Photometry of Haumea.

\begin{tabular}{|c|c|c|c|c|c|}
\hline UT Date $^{a}$ & Julian Date ${ }^{\mathrm{a}}$ & $m_{J}{ }^{\mathrm{b}}$ & UT Date $^{a}$ & Julian Date $^{\mathrm{a}}$ & $m_{J}{ }^{\mathrm{b}}$ \\
\hline 2008 Apr 15 & 2454571.609852 & 16.370 & 2008 Apr 1 & 2454571.760570 & $16.416 \pm 0.022$ \\
\hline 2008 Apr 15.11066 & 2454571.610665 & $16.352 \pm 0.022$ & 2008 Apr 15.26138 & 2454571.761382 & $16.441 \pm 0.022$ \\
\hline 2008 Apr 15.11175 & 2454571.611747 & $16.352 \pm 0.020$ & 2008 Apr 15.26790 & 2454571.767900 & $16.411 \pm 0.022$ \\
\hline 2008 Apr 15.11255 & 2454571.612546 & $16.338 \pm 0.024$ & 2008 Apr 15.26872 & 2454571.768716 & $16.398 \pm 0.022$ \\
\hline 2008 Apr 15.16761 & 2454571.667607 & $16.352 \pm 0.019$ & 2008 Apr 15.26979 & 2454571.769791 & $16.404 \pm 0.024$ \\
\hline 2008 Apr 15.16842 & 2454571.668420 & $16.334 \pm 0.019$ & 2008 Apr 15.27060 & 2454571.770602 & $16.371 \pm 0.023$ \\
\hline 2008 Apr 15.18420 & 2454571.684204 & $16.385 \pm 0.020$ & 2008 Apr 15.27696 & 2454571.776959 & $16.309 \pm 0.022$ \\
\hline 2008 Apr 15.18502 & 2454571.685020 & $16.367 \pm 0.019$ & 2008 Apr 15.27777 & 2454571.777775 & $16.260 \pm 0.022$ \\
\hline 2008 Apr 15.18610 & 2454571.686098 & $16.386 \pm 0.019$ & 2008 Apr 15.27886 & 2454571.778859 & $16.305 \pm 0.022$ \\
\hline 2008 Apr 15.18691 & 2454571.686910 & $16.390 \pm 0.019$ & 2008 Apr 15.27967 & 2454571.779671 & $16.272 \pm 0.022$ \\
\hline 2008 Apr 15.19373 & 2454571.693727 & $16.343 \pm 0.018$ & 2008 Apr 15.28660 & 2454571.786601 & $16.189 \pm 0.026$ \\
\hline 2008 Apr 15.19454 & 2454571.694539 & $16.340 \pm 0.019$ & 2008 Apr 15.28741 & 2454571.787407 & $16.173 \pm 0.022$ \\
\hline 2008 Apr 15.19562 & 2454571.695623 & $16.327 \pm 0.018$ & 2008 Apr 15.28849 & 2454571.788485 & $16.200 \pm 0.024$ \\
\hline 2008 Apr 15.19644 & 2454571.696435 & $16.332 \pm 0.018$ & 2008 Apr 15.28930 & 2454571.789298 & $16.186 \pm 0.024$ \\
\hline 2008 Apr 15.20279 & 2454571.702793 & $16.263 \pm 0.019$ & 2008 Apr 15.29585 & 2454571.795847 & $16.158 \pm 0.021$ \\
\hline 2008 Apr 15.20361 & 2454571.703606 & $16.234 \pm 0.019$ & 2008 Apr 15.29666 & 2454571.796665 & $16.132 \pm 0.021$ \\
\hline 2008 Apr 15.20468 & 2454571.704682 & $16.247 \pm 0.019$ & 2008 Apr 15.29774 & 2454571.797743 & $16.112 \pm 0.022$ \\
\hline 2008 Apr 15.20549 & 2454571.705494 & $16.237 \pm 0.019$ & 2008 Apr 15.29856 & 2454571.798556 & $16.121 \pm 0.021$ \\
\hline 2008 Apr 15.21199 & 2454571.711991 & $16.180 \pm 0.019$ & 2008 Apr 15.30507 & 2454571.805070 & $16.130 \pm 0.020$ \\
\hline 2008 Apr 15.21280 & 2454571.712804 & $16.174 \pm 0.019$ & 2008 Apr 15.30588 & 2454571.805884 & $16.135 \pm 0.021$ \\
\hline 2008 Apr 15.21390 & 2454571.713896 & $16.172 \pm 0.019$ & 2008 Apr 15.30697 & 2454571.806969 & $16.162 \pm 0.021$ \\
\hline 2008 Apr 15.21471 & 2454571.714708 & $16.186 \pm 0.019$ & 2008 Apr 15.30778 & 2454571.807784 & $16.123 \pm 0.020$ \\
\hline 2008 Apr 15.22109 & 2454571.721087 & $16.167 \pm 0.018$ & 2008 Apr 15.31249 & 2454571.812494 & $16.191 \pm 0.020$ \\
\hline 2008 Apr 15.22190 & 2454571.721900 & $16.182 \pm 0.017$ & 2008 Apr 15.31330 & 2454571.813304 & $16.174 \pm 0.021$ \\
\hline 2008 Apr 15.22297 & 2454571.722974 & $16.173 \pm 0.019$ & 2008 Apr 15.31831 & 2454571.818312 & $16.212 \pm 0.021$ \\
\hline 2008 Apr 15.22379 & 2454571.723786 & $16.198 \pm 0.019$ & 2008 Apr 15.31913 & 2454571.819128 & $16.246 \pm 0.019$ \\
\hline 2008 Apr 15.23047 & 2454571.730466 & $16.228 \pm 0.019$ & 2008 Apr 15.32385 & 2454571.823849 & $16.286 \pm 0.019$ \\
\hline 2008 Apr 15.23128 & 2454571.731280 & $16.252 \pm 0.019$ & $2008 \mathrm{Apr} 15.32466$ & 2454571.824657 & $16.301 \pm 0.021$ \\
\hline 2008 Apr 15.23236 & 2454571.732358 & $16.232 \pm 0.020$ & 2008 Apr 15.32953 & 2454571.829527 & $16.330 \pm 0.025$ \\
\hline 2008 Apr 15.23317 & 2454571.733171 & $16.261 \pm 0.019$ & 2008 Apr 15.33034 & 2454571.830342 & $16.318 \pm 0.022$ \\
\hline 2008 Apr 15.23952 & 2454571.739521 & $16.333 \pm 0.021$ & 2008 Apr 15.33506 & 2454571.835055 & $16.351 \pm 0.022$ \\
\hline 2008 Apr 15.24034 & 2454571.740339 & $16.336 \pm 0.022$ & 2008 Apr 15.33586 & 2454571.835859 & $16.361 \pm 0.021$ \\
\hline 2008 Apr 15.24140 & 2454571.741404 & $16.350 \pm 0.021$ & 2008 Apr 15.34632 & 2454571.846321 & $16.375 \pm 0.022$ \\
\hline 2008 Apr 15.24222 & 2454571.742216 & $16.345 \pm 0.020$ & 2008 Apr 15.34713 & 2454571.847132 & $16.377 \pm 0.020$ \\
\hline 2008 Apr 15.24959 & 2454571.749588 & $16.392 \pm 0.020$ & 2008 Apr 15.34822 & 2454571.848217 & $16.382 \pm 0.021$ \\
\hline 2008 Apr 15.25039 & 2454571.750392 & $16.425 \pm 0.020$ & 2008 Apr 15.34911 & 2454571.849106 & $16.352 \pm 0.020$ \\
\hline 2008 Apr 15.25147 & 2454571.751474 & $16.432 \pm 0.020$ & 2008 Apr 15.35012 & 2454571.850125 & $16.347 \pm 0.020$ \\
\hline 2008 Apr 15.25229 & 2454571.752288 & $16.415 \pm 0.021$ & 2008 Apr 15.35094 & 2454571.850936 & $16.366 \pm 0.020$ \\
\hline 2008 Apr 15.25867 & 2454571.758673 & $16.420 \pm 0.022$ & 2008 Apr 15.35202 & 2454571.852017 & $16.366 \pm 0.020$ \\
\hline 2008 Apr 15.25949 & 2454571.759489 & $16.409 \pm 0.024$ & 2008 Apr 15.35283 & 2454571.852828 & $16.347 \pm 0.020$ \\
\hline
\end{tabular}

a Dates in Haumea's reference frame;

b Apparent magnitude.

\section{RESULTS AND DISCUSSION}

\subsection{Color Versus Rotation}

The new data record just over one full rotation of Haumea. Figure 1 combines previously published $B$ and $R$ data (Lacerda et al. 2008) with the new $J$ and $H$ data and shows that all four filters exhibit very similar variability with a combined total range $\Delta m=0.30 \pm 0.02$ mag. As described in $\$ 2$, to improve the signal-to-noise ratios of the $J$ and $H$ data, we binned sets of measurements taken back-to-back (usually sets of four); the resulting lightcurve is shown in Fig. 2. There, the previously identified dark, red spot (DRS; Lacerda et al. 2008) on the surface of Haumea is clearly apparent at rotational phases close to $\phi=0.8$ in the $B$ and $R$ curves. The nearinfrared data generally follow the $R$-band data but show a slight visible/near-infrared reddening which coincides with the DRS.

The differences between the individual lightcurves in Figs. 1 and 2 are small. To highlight color variations on Haumea, we plot in Fig. 3 all possible combinations of visible-to-near-infrared color curves. The curves are calculated by interpolating the better-sampled $B$ and $R$ data to the binned $J$ and $H$ rotational phases (Fig. (2) and subtracting. The error bars are dominated by the uncertainties in the near-infrared measurements, which are added quadratically to the mean $B$ or $R$ errors. When taken separately, the color curves in Fig. 3 appear to differ only marginally from a rotationally constant value. However, the color $B-H$, and arguably $B-J, R-H$, and $J-H$, show visible reddening humps for rotational phases close to where the DRS was found to lie $(\phi \sim 0.8)$. To locate and quantify color variability features in the curves in Fig. 3 we employ a running Gaussian probability test. In this test we consider a moving rotational phase window and calculate the quantity

$$
G=\frac{\sum_{i=1}^{N}\left(c_{i}-c_{0}\right) / e_{i}}{\sqrt{N}}
$$

for the points that fall within the window. In Eq. (1), $N$ is the number of points within the window, $c_{i}$ and $e_{i}$ are the color values and respective error bars of those points, and $c_{0}$ is the median color of all points (dotted horizontal lines in Fig. 31). We then move the window along each color curve in rotational phase steps of 0.05 to obtain a running- $G$ value. Equation (11) represents a Gaussian deviate with zero mean and unity standard deviation and 
TABLE 3

$H$-band Photometry of Haumea.

\begin{tabular}{|c|c|c|c|c|c|}
\hline UT Date $^{a}$ & Julian Date ${ }^{a}$ & $m_{H} \mathrm{~b}$ & UT Date $^{a}$ & Julian Date ${ }^{a}$ & $m_{H} \mathrm{~b}$ \\
\hline 2008 Apr 1 & 4571.614839 & 16. & 2008 Apr 1 & 2454571.754303 & $16.505 \pm 0.024$ \\
\hline 2008 Apr 15.11553 & 2454571.615528 & $16.396 \pm 0.026$ & 2008 Apr 15.25501 & 2454571.755009 & $16.493 \pm 0.022$ \\
\hline 2008 Apr 15.11648 & 2454571.616483 & $16.355 \pm 0.024$ & 2008 Apr 15.25596 & 2454571.755956 & $16.489 \pm 0.022$ \\
\hline 2008 Apr 15.11719 & 2454571.617187 & $16.382 \pm 0.025$ & 2008 Apr 15.25665 & 2454571.756649 & $16.474 \pm 0.022$ \\
\hline 2008 Apr 15.17144 & 2454571.671445 & $16.449 \pm 0.021$ & 2008 Apr 15.26340 & 2454571.763400 & $16.478 \pm 0.022$ \\
\hline 2008 Apr 15.17213 & 2454571.672129 & $16.423 \pm 0.020$ & 2008 Apr 15.26409 & 2454571.764093 & $16.490 \pm 0.026$ \\
\hline 2008 Apr 15.17311 & 2454571.673107 & $16.426 \pm 0.021$ & 2008 Apr 15.26505 & 2454571.765051 & $16.527 \pm 0.025$ \\
\hline 2008 Apr 15.17380 & 2454571.673799 & $16.424 \pm 0.021$ & 2008 Apr 15.26574 & 2454571.765742 & $16.500 \pm 0.026$ \\
\hline 2008 Apr 15.18920 & 2454571.689196 & $16.430 \pm 0.021$ & 2008 Apr 15.27262 & 2454571.772622 & $16.388 \pm 0.025$ \\
\hline 2008 Apr 15.18989 & 2454571.689885 & $16.402 \pm 0.021$ & 2008 Apr 15.27332 & 2454571.773318 & 16.455 \\
\hline 2008 Apr 15.19086 & 2454571.690862 & $16.414 \pm 0.022$ & 2008 Apr 15.27427 & 2454571.774272 & $16.432 \pm 0.031$ \\
\hline 2008 Apr 15.19155 & 2454571.691552 & $16.413 \pm 0.022$ & 2008 Apr 15.27496 & 2454571.774963 & $16.401 \pm 0.027$ \\
\hline 2008 Apr 15.19847 & 2454571.698466 & $16.349 \pm 0.019$ & 2008 Apr 15.28168 & 2454571.781679 & 16.273 \\
\hline 2008 Apr 15.19915 & 2454571.699147 & $16.340 \pm 0.020$ & 2008 Apr 15.28237 & 2454571.782369 & $16.333 \pm 0.033$ \\
\hline 2008 Apr 15.20011 & 2454571.700109 & $16.335=$ & 2008 Apr 15.28332 & 2454571.783321 & 16.30 \\
\hline 2008 Apr 15.20079 & 2454571.700787 & $16.330 \pm 0.017$ & 2008 Apr 15.28401 & 2454571.784011 & $16.290 \pm 0.034$ \\
\hline 2008 Apr 15.20749 & 2454571.707494 & $16.261 \pm 0.019$ & 2008 Apr 15.29131 & 2454571.791314 & $16.249 \pm 0.024$ \\
\hline 2008 Apr 15.20819 & 2454571.708186 & $16.268 \pm 0.020$ & 2008 Apr 15.29201 & 2454571.792008 & $16.210 \pm 0.023$ \\
\hline 2008 Apr 15.20914 & 2454571.709144 & $16.233 \pm 0.020$ & 2008 Apr 15.29298 & 2454571.792983 & $16.188 \pm 0.021$ \\
\hline 2008 Apr 15.20984 & 2454571.709836 & $16.225 \pm 0.020$ & 2008 Apr 15.29367 & 2454571.793674 & $16.165 \pm 0.022$ \\
\hline 2008 Apr 15.21673 & 2454571.716730 & $16.238 \pm 0.018$ & 2008 Apr 15.30058 & 2454571.800584 & $16.186 \pm 0.020$ \\
\hline 2008 Apr 15.21742 & 2454571.717424 & $16.197 \pm 0.018$ & 2008 Apr 15.30126 & 2454571.801264 & $16.192 \pm 0.019$ \\
\hline 2008 Apr 15.21838 & 2454571.718383 & $16.235 \pm 0.019$ & 2008 Apr 15.30222 & 2454571.802219 & $16.181 \pm 0.020$ \\
\hline 2008 Apr 15.21907 & 2454571.719073 & 0.019 & 2008 Apr 15.30291 & 2454571.802912 & $16.179 \pm 0.021$ \\
\hline 2008 Apr 15.22582 & 2454571.725818 & $16.246 \pm 0.019$ & 2008 Apr 15.30979 & 2454571.809794 & $16.213 \pm 0.023$ \\
\hline 2008 Apr 15.22651 & 2454571.726511 & $16.245 \pm 0.020$ & 2008 Apr 15.31049 & 2454571.810488 & $16.220 \pm 0.023$ \\
\hline 2008 Apr 15.22747 & 2454571.727468 & $16.261 \pm 0.019$ & 2008 Apr 15.31532 & 2454571.815318 & $16.253 \pm 0.024$ \\
\hline 2008 Apr 15.22816 & 2454571.728161 & $16.255 \pm 0.019$ & 2008 Apr 15.31601 & 2454571.816013 & $16.253 \pm 0.023$ \\
\hline 2008 Apr 15.23518 & 2454571.735181 & 0.021 & 2008 Apr 15.32114 & 2454571 . & $16.333 \pm 0.024$ \\
\hline 2008 Apr 15.23588 & 2454571.735878 & $16.344 \pm 0.020$ & 2008 Apr 15.32183 & 2454571.821831 & $16.287 \pm 0.024$ \\
\hline 2008 Apr 15.23683 & 2454571.736832 & $16.369 \pm 0.023$ & 2008 Apr 15.32668 & 2454571.826679 & $16.383 \pm 0.026$ \\
\hline 2008 Apr 15.23751 & 2454571.737510 & $16.343 \pm 0.021$ & 2008 Apr 15.32736 & 2454571.827364 & $16.389 \pm 0.025$ \\
\hline 2008 Apr 15.24423 & 2454571.744229 & $16.394 \pm 0.023$ & 2008 Apr 15.33236 & 2454571.832360 & $16.457 \pm 0.028$ \\
\hline 2008 Apr 15.24492 & 2454571.744916 & $16.408 \pm 0.024$ & 2008 Apr 15.33305 & 2454571.833051 & $16.393 \pm 0.026$ \\
\hline 2008 Apr 15.24587 & 2454571.745869 & $16.428 \pm 0.023$ & 2008 Apr 15.33789 & 2454571.837886 & $16.443 \pm 0.028$ \\
\hline 2008 Apr 15.24656 & 2454571.746561 & $16.459 \pm 0.023$ & 2008 Apr 15.33861 & 2454571.838607 & $16.439 \pm 0.027$ \\
\hline
\end{tabular}

${ }^{a}$ Dates in Haumea's reference frame;

b Apparent magnitude.

TABLE 4

BinNED $J$-BAND Data.

\begin{tabular}{lcc}
\hline \hline Mean UT Date $^{\mathrm{a}}$ & Mean Julian Date $^{\mathrm{a}}$ & ${\text { Mean } m_{J}^{\mathrm{b}}}^{\mathrm{b}}$ \\
\hline 2008 Apr 15.11120 & 2454571.611202 & $16.353 \pm 0.013$ \\
2008 Apr 15.16801 & 2454571.668013 & $16.343 \pm 0.013$ \\
2008 Apr 15.18556 & 2454571.685558 & $16.382 \pm 0.010$ \\
2008 Apr 15.19508 & 2454571.695081 & $16.336 \pm 0.009$ \\
2008 Apr 15.20414 & 2454571.704144 & $16.245 \pm 0.010$ \\
2008 Apr 15.21335 & 2454571.713350 & $16.178 \pm 0.009$ \\
2008 Apr 15.22244 & 2454571.722437 & $16.180 \pm 0.010$ \\
2008 Apr 15.23182 & 2454571.731819 & $16.243 \pm 0.011$ \\
2008 Apr 15.24087 & 2454571.740870 & $16.341 \pm 0.010$ \\
2008 Apr 15.25094 & 2454571.750936 & $16.416 \pm 0.012$ \\
2008 Apr 15.26003 & 2454571.760028 & $16.422 \pm 0.012$ \\
2008 Apr 15.26925 & 2454571.769252 & $16.396 \pm 0.013$ \\
2008 Apr 15.27832 & 2454571.778316 & $16.287 \pm 0.015$ \\
2008 Apr 15.28795 & 2454571.787948 & $16.187 \pm 0.012$ \\
2008 Apr 15.29720 & 2454571.797203 & $16.131 \pm 0.013$ \\
2008 Apr 15.30643 & 2454571.806427 & $16.138 \pm 0.012$ \\
2008 Apr 15.31290 & 2454571.812899 & $16.183 \pm 0.014$ \\
2008 Apr 15.31872 & 2454571.818720 & $16.229 \pm 0.018$ \\
2008 Apr 15.32425 & 2454571.824253 & $16.294 \pm 0.013$ \\
2008 Apr 15.32993 & 2454571.829934 & $16.324 \pm 0.015$ \\
2008 Apr 15.33546 & 2454571.835457 & $16.356 \pm 0.013$ \\
2008 Apr 15.34769 & 2454571.847694 & $16.372 \pm 0.011$ \\
2008 Apr 15.35148 & 2454571.851476 & $16.357 \pm 0.010$ \\
& & \\
\hline
\end{tabular}

a Dates in Haumea's reference frame;

b Mean apparent magnitude.
TABLE 5

Binned $H$-BAND Data.

\begin{tabular}{lcc}
\hline \hline Mean UT Date $^{\mathrm{a}}$ & Mean Julian Date $^{\mathrm{a}}$ & Mean $m_{H}{ }^{\mathrm{b}}$ \\
\hline 2008 Apr 15.11601 & 2454571.616009 & $16.376 \pm 0.015$ \\
2008 Apr 15.17262 & 2454571.672620 & $16.431 \pm 0.011$ \\
2008 Apr 15.19037 & 2454571.690374 & $16.415 \pm 0.011$ \\
2008 Apr 15.19963 & 2454571.699627 & $16.339 \pm 0.009$ \\
2008 Apr 15.20866 & 2454571.708665 & $16.247 \pm 0.013$ \\
2008 Apr 15.21790 & 2454571.717903 & $16.220 \pm 0.012$ \\
2008 Apr 15.22699 & 2454571.726990 & $16.252 \pm 0.009$ \\
2008 Apr 15.23635 & 2454571.736350 & $16.352 \pm 0.011$ \\
2008 Apr 15.24539 & 2454571.745394 & $16.423 \pm 0.016$ \\
2008 Apr 15.25548 & 2454571.755479 & $16.491 \pm 0.012$ \\
2008 Apr 15.26457 & 2454571.764572 & $16.499 \pm 0.014$ \\
2008 Apr 15.27379 & 2454571.773793 & $16.419 \pm 0.018$ \\
2008 Apr 15.28284 & 2454571.782845 & $16.300 \pm 0.018$ \\
2008 Apr 15.29249 & 2454571.792495 & $16.203 \pm 0.019$ \\
2008 Apr 15.30174 & 2454571.801745 & $16.185 \pm 0.009$ \\
2008 Apr 15.31014 & 2454571.810141 & $16.217 \pm 0.014$ \\
2008 Apr 15.31567 & 2454571.815666 & $16.253 \pm 0.013$ \\
2008 Apr 15.32148 & 2454571.821483 & $16.310 \pm 0.023$ \\
2008 Apr 15.32702 & 2454571.827022 & $16.386 \pm 0.015$ \\
2008 Apr 15.33271 & 2454571.832706 & $16.425 \pm 0.030$ \\
2008 Apr 15.33825 & 2454571.838247 & $16.441 \pm 0.016$ \\
& & \\
\hline
\end{tabular}

a Dates in Haumea's reference frame;

b Mean apparent magnitude. 
TABLE 6

MeAN MAGNITUdes AND COLORS.

\begin{tabular}{cccccc}
\hline \hline Object & $m_{J}{ }^{\mathrm{a}}$ & $m_{H}{ }^{\mathrm{b}}$ & $B-R$ & $R-J$ & $J-H$ \\
\hline Haumea & $16.29 \pm 0.09$ & $16.34 \pm 0.10$ & $0.972 \pm 0.014$ & $0.885 \pm 0.012$ & $-0.057 \pm 0.016$ \\
Satellite & $18.92 \pm 0.09$ & $19.32 \pm 0.10$ & $\cdots$ & $\cdots$ & $-0.399 \pm 0.034$
\end{tabular}

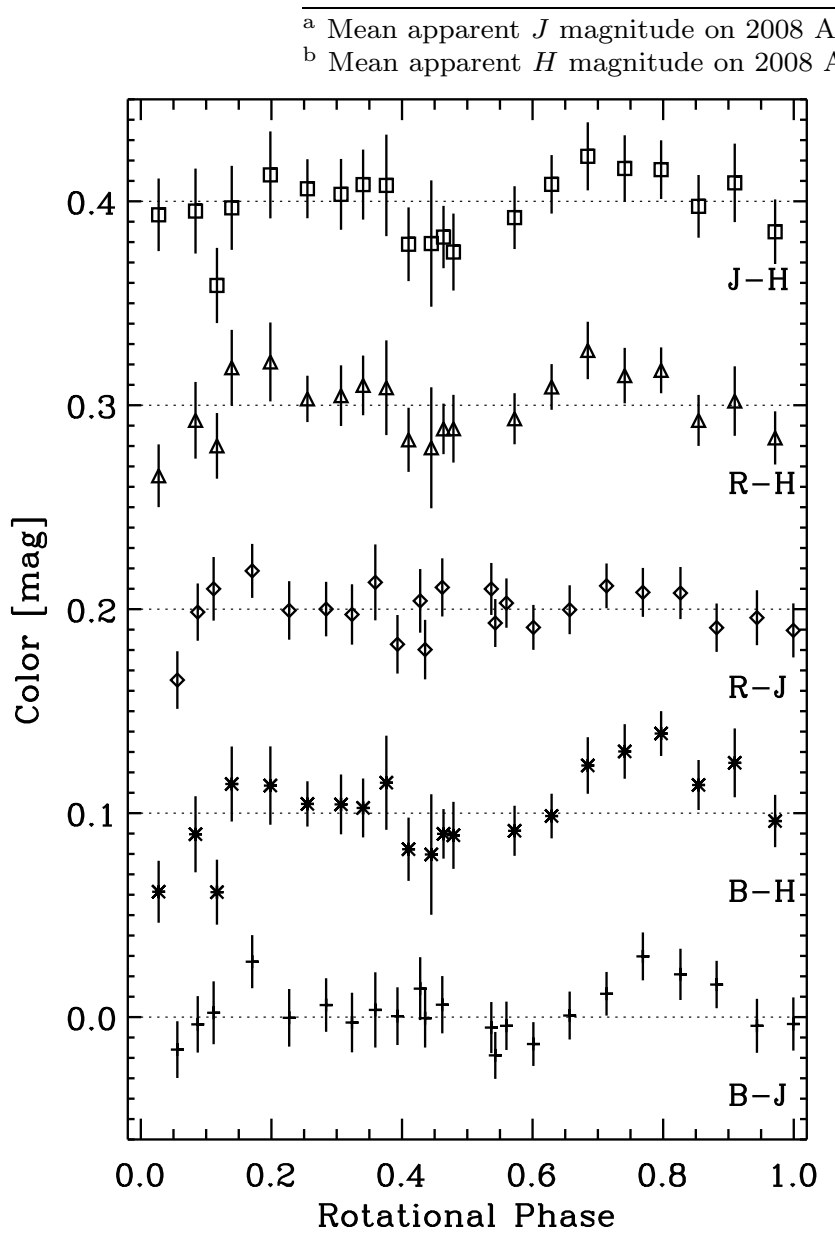

FIG. 3.- Visible and near-infrared color curves of Haumea. Each curve represents deviations from the respective rotationallymedianed mean color (from bottom up, $B-J=1.856 \pm 0.012$ mag, $B-H=1.799 \pm 0.021 \mathrm{mag}, R-J=0.885 \pm 0.012 \mathrm{mag}$, $R-H=0.828 \pm 0.016 \mathrm{mag}$, and $J-H=-0.057 \pm 0.016 \mathrm{mag})$ and is vertically shifted by $0.1 \mathrm{mag}$ for clarity. Slight reddening bumps are observable in $B-H, B-J$, and possibly in $R-H$ and $J-H$.

can thus be converted to a Gaussian probability, $p(G)$, assuming that the points are normally distributed around the median. The probability $p(G)$ is sensitive to unlikely sequences of deviant points, all on one side of the median. Figure 4 shows the test results for each of the color curves using a window size $\Delta \phi=0.25$ (see discussion below). The Figure shows that the $B-H$ curve has a significant $(\sim 4 \sigma)$ non-random feature close to $\phi=0.8$. The test also detects weaker $(2.8 \sigma$ and $2.5 \sigma)$ features in the $B-J$ and $R-H$ curves close to $\phi=0.8$.

The size of the rotational phase window $\Delta \phi$ is physically motivated by the fraction of the surface of Haumea that is visible at any given instant. In that sense, it should not be larger than $\Delta \phi=0.5$. Moreover, although

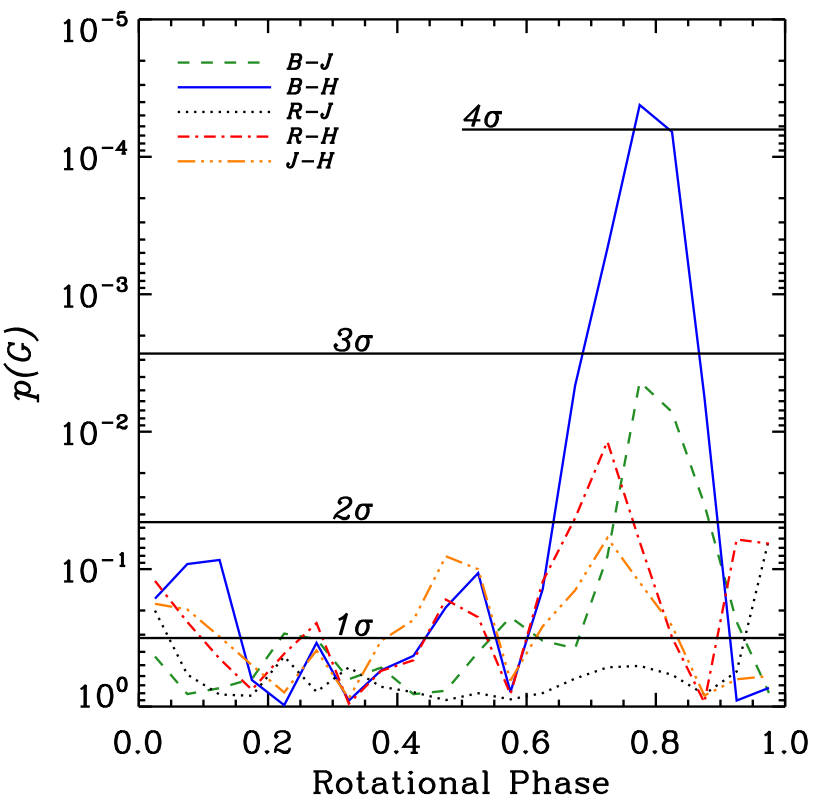

FIG. 4.- Running Gaussian probability for each of the color curves in Fig. 3 Unlikely sequences of points all above or below the median colors (horizontal, dotted lines in Fig. 3) will be visible as peaks in this Figure. The color curve $B-H$ color shows a significant peak at rotational phase $\phi \sim 0.8$. Curves $B-J$ and $R-H$ show smaller but noticeable features at the same rotational phase.

half the surface is visible, projection effects in the limb region will make the effective visible area smaller, by possibly another factor 2. In Fig. 5 we illustrate the effect of the window size by replotting the running $p(G)$ for color curve $B-H$ using four window sizes, $\Delta \phi=0.20,0.25$, 0.33 , and 0.50 . As expected, $p(G)$ does not differ much for windows $0.20 \leq \Delta \phi \leq 0.33$. For the largest window size $\Delta \phi=0.50$ the probability begins to appear dilluted, but even then the test succeeds in locating the feature at $\phi=0.8$.

The near-infrared measurements presented here are considerably less numerous than the the optical data that were used to identify the DRS in $B-R$ (Lacerda et al. 2008). Also, the $J$ and $H$ measurements may show systematic correlations because they were measured on the same night using the same telescope. Nevertheless, the observed changes in $B$ and $R$ relative to $J$ and $H$ do not suffer from this effect and are likely to be real. A visible/near-infrared reddening was already observed in our 2007 data (see $J_{2007}$ points in Fig. 1) adding confidence to our conclusions. The results presented above suggest that the region close to the DRS is also spectrally anomalous in the visible-to-near-infrared wavelength range with respect to the average surface of Haumea. 


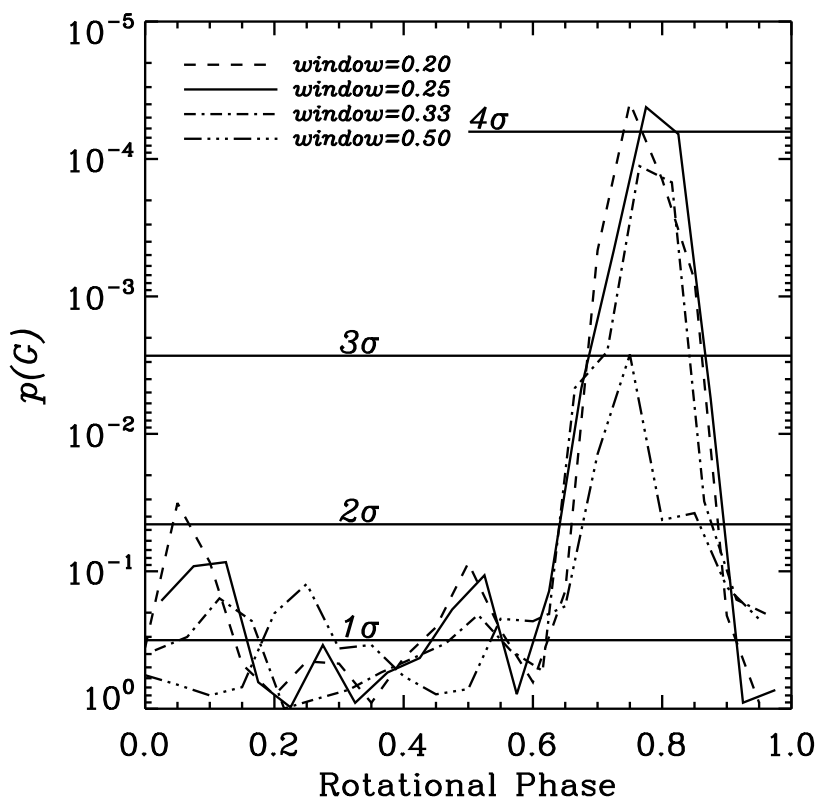

FIG. 5.- Running Gaussian probability for the $B-H$ color curve for four window sizes, $\Delta \phi=0.20,0.25,0.33$, and 0.50 . The running probability $p(G)$ is calculated in a window of width $\Delta \phi$ which is evaluated in rotational phase steps of 0.05 . The differences are minimal for window sizes $0.20 \leq \Delta \phi<0.33$. For the largest window size $\Delta \phi=0.50$ the probability begins to appear dilluted. See text for details.

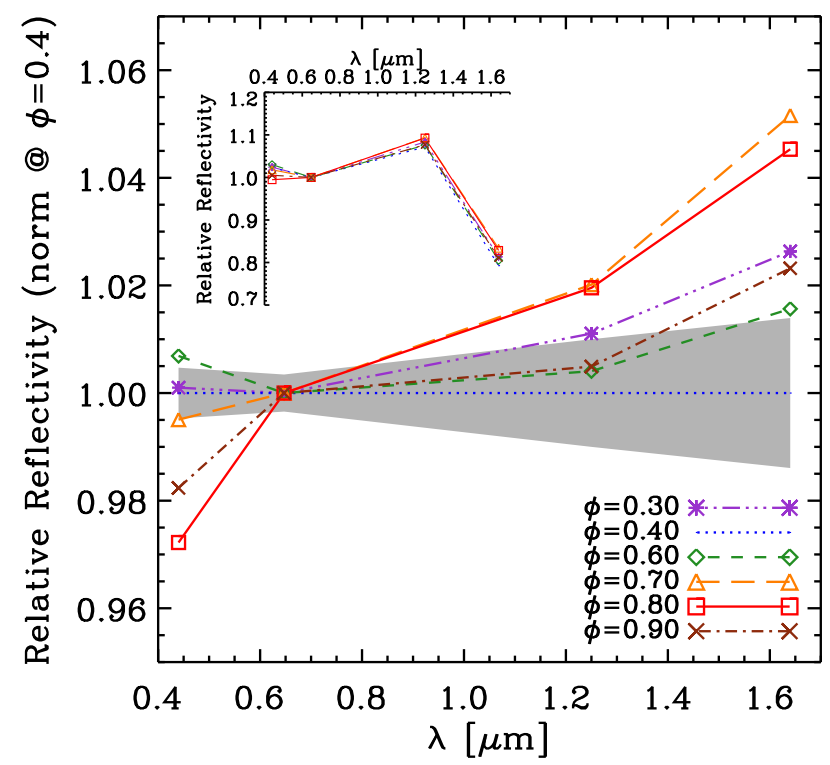

FIG. 6.- Normalized reflectivity vs. wavelength for different rotational phases. The reflectivities were computed from the broadband colors by subtracting the colors of the Sun $(B-R=0.996$, $R-J=0.797, J-H=0.258$; cf. Holmberg et al. 2006). All curves were normalized at the $R$-band, and divided by the curve at rotational phase $\phi=0.4$, for clarity. The inset shows the reflectivities prior to dividing by the $\phi=0.4$ curve. The shaded polygon represents the uncertainty propagated from errors of measurement.

In Fig. 6] we combine our four-band data to produce reflectivity vs. wavelength curves at different rotational phases. We focus on the DRS region and plot curves at $\phi=0.3$ and $\phi=0.4$ as illustrative of the mean Haumea surface. We employed interpolation to calculate color indices at the given rotational phases, which were sub- sequently converted to reflectivities relative to the $R$ band. To enhance the subtle differences with rotation we normalize all curves by that at $\phi=0.4$; an inset in Fig. 6] shows the reflectivities before normalization. Figure 6 shows that relative to the majority of the surface of Haumea the region near $\phi=0.7$ displays an enhanced $H$ band reflectivity which, close to $\phi=0.8$, is accompanied by a depressed $B$-band reflectivity. Close to $\phi=0.9, B$ remains depressed, while $H$ is restored to average values. We note that this variation is consistent with the results shown in Fig. 4. To summarize, the DRS region is both fainter in $B$ and brighter in $H$ than the rest of Haumea.

The presence of a blue absorber on the DRS could explain the fainter $B$ reflectance. A recent $U$ - and $B$ band photometric study of KBOs (Jewitt et al. 2007) suggests that objects in the classical population (objects in quasi-circular orbits between the 2:1 and the 3:2 meanmotion resonance with Neptune) lack significant blue absorption. As discussed by those authors, $B$-band absorption in main-belt asteroids is generally linked with the presence of phyllosilicates and other hydrated minerals (see also Gaffey \& McCord 1978; Feierberg et al. 1985) and is a characteristic feature in the spectra of $\mathrm{C}$ and G-type asteroids (Tholen \& Barucci 1989). How likely is it that Haumea has hydrated minerals on its surface? Although Haumea is located in the classical population as defined above, it is atypical in its waterice dominated surface spectrum and its high bulk density (2.5 times water). These two properties suggest a differentiated body with significant rocky content. Furthermore, KBOs (mainly the larger ones) have possibly sustained liquid water in their interiors due to radiogenic heating (Merk \& Prialnik 2006). It would therefore be unremarkable to find trace amounts of hydrated minerals on Haumea. In fact, fits to the near-infrared spectrum of Haumea are slightly improved by the addition of phyllosilicates such as kaolinite and montmorillonite (Truijllo et al. 2007). Ultraviolet spectra of Haumea at different rotational phases are needed to further constrain the character of the blue absorption close to the DRS.

Our $J$ and $H$ photometry suggests that the $1.5 \mu \mathrm{m}$ water-ice band is weaker (less deep) close to the DRS. In contrast, Lacerda et al. (2008) found marginal evidence that the $1.5 \mu \mathrm{m}$ band is deeper close to the DRS. One difference between the two measurements is that while here we use $J$ vs. $H$, Lacerda et al. (2008) used $J$ vs. the "CH4 $4_{s}$ filter to assess possible variations in the waterice band. The latter filter has a bandpass (center 1.60 $\mu \mathrm{m}$, FWHM $0.11 \mu \mathrm{m}$ ) between the $1.5 \mu \mathrm{m}$ and the 1.65 $\mu \mathrm{m}$ band diagnostic of crystalline water-ice and is thus affected by the degree of crystallinity of the ice. The two measurements can be reconciled if the DRS material has an overall less deep $1.5 \mu \mathrm{m}$ water-ice band but a larger relative abundance of crystalline water ice. We simulated this scenario using synthetic reflectance spectra [calculated using published optical constants for crystalline water ice (Grundy \& Schmitt 1998) and a Hapke model with the best fit parameters for Haumea (Trujillo et al. 2007)]. By convolving two model spectra, one for $T=30$ $\mathrm{K}$ and one for $T=140 \mathrm{~K}$ (to simulate a weaker crystalline band), with the $J, H$, and $\mathrm{CH}_{s}$ bandpasses we found an effect similar to what is observed. The $30 \mathrm{~K}$ spectrum, taken to represent the DRS material, shows a 


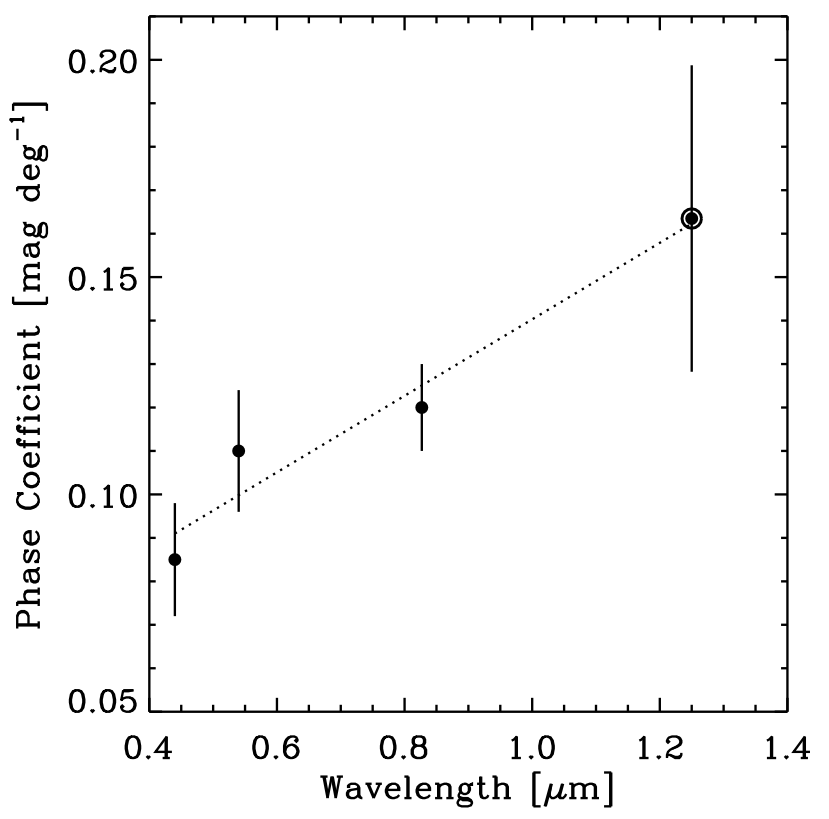

FIG. 7.- Phase coefficient of Haumea as a function of wavelength. From left to right, points mark the central wavelength of the $B, V$, $I$, and $J$ bands. Our measurement of the $J$-band phase coefficient, $\beta_{J}=0.16 \pm 0.04 \mathrm{mag} \mathrm{deg}^{-1}$ is highlighted. The remaining three points are taken from Rabinowitz et al. (2006). A linear best-fit (slope 0.09 and $1 \mu \mathrm{m}$ value 0.14 ) is overplotted as a dotted line.

$\sim 3 \%$ higher $H$-to- $J$ flux ratio, but a $\sim 4 \%$ lower $\mathrm{CH}_{s}$-to- $J$ flux ratio than the $140 \mathrm{~K}$ spectrum. However, this possibility is not unique and time-resolved $H$-band spectra are required to test this scenario.

\subsection{Phase Function}

Atmosphereless solar system bodies exhibit a linear increase in brightness with decreasing phase angle. At small angles $(\alpha<0.01$ to $1 \mathrm{deg})$, this phase function becomes non-linear causing a sharp magnitude peak. The main physical mechanisms thought to be responsible for this opposition brightening effect are shadowing and coherent backscattering. In simple terms, shadowing occurs because although a photon can always scatter back in the direction from which it hit the surface, other directions may be blocked. The implication is that backilluminated $\left(\alpha \sim 0^{\circ}\right)$ objects do not shadow their own surfaces and appear brighter. The brightening due to coherent backscattering results from the constructive interference of photons that scatter in the backwards direction from pairs of surface particles (or of features within a particle; Nelson et al. 2000). The constructive interfence decreases rapidly with increasing phase angle. Generally, it is believed that shadowing regulates the decrease in brightness with phase angle from a few up to tens of degrees, while coherent backscattering mainly produces the near-zero phase angle spike (French et al. 2007).

The relative importance of shadowing and coherent backscattering on a given surface is difficult to assess. An early prediction by Hapke (1993) was that the angular width of the exponential brightness peak should vary linearly with wavelength in the case of coherent backscattering, given its interference nature. Shadowing, on the other hand, should be acromatic. However, more recent work has shown that the wavelength dependence of coherent backscattering can be very weak

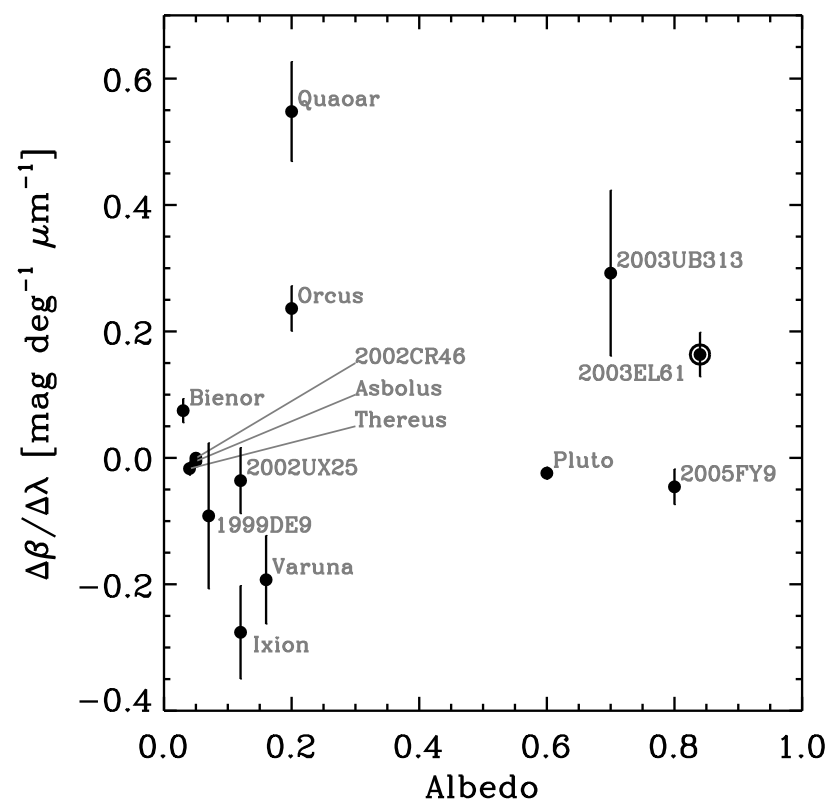

FIG. 8. - Slope of the $\beta(\lambda)$ relation versus approximate geometric albedo for a number of KBOs and Centaurs. No clear relation exists between the two quantities.

(Nelson et al. 2000). Thus, while a strong wavelength dependence is usually attributable to coherent backscattering, a weak wavelength dependence may be explained by either mechanism. It was also expected that higher albedo surfaces should be less affected by shadowing because more multiply scattered photons will reach the observer even from shadowed surface regions (Nelson et al. 1998). Coherent backscattering is a multiple-scattering process that thrives on highly reflective surfaces. Subsequent studies have shown that low albedo surfaces can also display strong coherent backscattering at very low phase angles (Hapke et al. 1998). Finally, while both effects cause a brightening towards opposition, only coherent backscattering has an effect on the polarization properties of light scattered from those objects: it favors the electric field component parallel to the scattering plane and thus gives rise to partially linearly polarized light (Boehnhardt et al. 2004; Bagnulo et al. 2006). With the currently available instruments, useful polarization measurements can only be obtained on the brightest KBOs.

Our July $2007 J$-band lightcurve data (Lacerda et al. 2008) and the April 2008 data presented here together show that Haumea appears brighter in the latter dataset. After calculating the time-medianed apparent magnitudes, and correcting them to unit helio- and geocentric distances $(R$ and $\Delta)$ using $m_{J}(1,1, \alpha)=m_{J}-5 \log (R \Delta)$, a difference of 0.096 magnitudes remains between the datasets, which we attribute to the different phase angles $\left(\alpha_{2007}=1.13 \mathrm{deg}\right.$ and $\left.\alpha_{2008}=0.55 \mathrm{deg}\right)$ at the two epochs. Assuming a phase function of the form $m_{J}(\alpha)=$ $m_{J}(1,1,0)+\beta_{J} \alpha$, we derive a slope $\beta_{J}=0.16 \pm 0.04 \mathrm{mag}$ $\operatorname{deg}^{-1}$. The uncertainty in $\beta_{J}$ was calculated by interpolating the $2008 \mathrm{~J}$ lightcurve to the rotational phases of the $2007 J$ lightcurve (both corrected to $R=\Delta=1 \mathrm{AU}$ ) and calculating the standard deviation of the difference.

In Fig. 7 we plot the phase coefficient $\beta$ versus the central wavelengths of the filters $B, V, I$, and $J$. The visible $\beta_{B, V, I}$ values are taken from Rabinowitz et al. 
(2006) where they are measured in the phase range $0.5<\alpha<1.0 \mathrm{deg}$. A linear fit to the relation is overplotted as a dotted line showing that $\beta_{\lambda}$ increases with $\lambda$. The fit has a slope $\Delta \beta / \Delta \lambda=0.09 \pm 0.03 \mathrm{mag} \mathrm{deg}^{-1}$ $\mu \mathrm{m}^{-1}$ and a $1 \mu \mathrm{m}$ value $\beta_{1 \mu \mathrm{m}}=0.14 \pm 0.02 \mathrm{mag} \mathrm{deg}^{-1}$. Using a $\chi^{2}$ test we are only able to reject a constant $\beta_{\lambda}$ at the $2 \sigma$ level. However, the monotonic increase of $\beta_{\lambda}$ with wavelength plus the high albedo $\left(p_{V}>0.6\right.$, Rabinowitz et al. 2006) of Haumea both suggest that coherent backscattering is dominant in the range of phase angles observed.

Small icy bodies in the solar system show very diverse phase function vs. wavelength behaviors (Rabinowitz et al. 2007). Most KBOs for which the phase curve has been measured in more than one band show steeper slopes towards longer wavelengths. We show here that in the case of Haumea this behavior extends into the near-infrared. The Centaurs (Rabinowitz et al. 2007) and the Uranian satellites (Karkoschka 2001) show little variation in the phase curve with wavelength for $\alpha<3$ to $6 \mathrm{deg}$, suggesting that shadowing is dominant over coherent backscattering. Other objects show opposite or even non-monotonic relations between phase function slope and wavelength. For instance, some type of terrain on the Jovian moon Europa have phase functions that vary nonmonotonically with wavelength (Helfenstein et al. 1998), while Pluto's phase function has a weak wavelength dependence opposite to that seen in Haumea, from $\beta_{B}=0.037 \pm 0.001 \mathrm{mag} \operatorname{deg}^{-1}$ to $\beta_{R}=0.032 \pm 0.001$ mag deg ${ }^{-1}$ (Buratti et al. 2003).

In Fig. 8 we plot the slope of the $\beta_{\lambda}$ vs. $\lambda$ relation against approximate geometric albedo for a number of KBOs and Centaurs. The values $\Delta \beta / \Delta \lambda$ were obtained from linear fits to the phase slope measurements in three bands by Rabinowitz et al. (2007). The scatter in Fig. 8 is substantial and no clear relation is evident between albedo and the wavelength dependance of the phase function. As a result, the interpretation of these results in terms of physical properties of the surface is problematic. The photometric (and polarimetric) phase functions depend in a non-trivial way on the size and spatial arrangement of surface regolith particles, as well as on their composition. Besides, the phase functions of KBOs can only be measured in a narrow range of phase angles (currently $0.5<\alpha<1.2 \mathrm{deg}$ in the case of Haumea) making it difficult to recognize the presence or width of narrow opposition peaks. Nevertheless, further evidence that coherent backscattering is responsible for the observed linearity between $\beta$ and $\lambda$ can be sought using polarization measurements of the surface of Haumea.

\subsection{Satellite}

We stacked our $\sim 70$ frames in each filter to increase the signal-to-noise ratio of any real features around Haumea and so attempt to detect the satellites. In the stacked frames, we used a field star as representative of the pointspread function (PSF), scaled it to the brightest pixel of Haumea and subtracted it from the KBO. The result is shown in Figs. 9 and 10, where the original image, the PSF, and the subtracted image are plotted; one satellite, Hi'iaka, is clearly visible through both filters. We measure a Hi'iaka-Haumea separation $d=0.872 \pm 0.002^{\prime \prime}$
$(\mathrm{PA}=184.67 \pm 0.13 \mathrm{deg})$, and flux ratios (with respect to Haumea) of $0.088 \pm 0.001$ and $0.064 \pm 0.002$ in $J$ and $H$, respectively. The mean UT of the frame stack is 2008 Apr 15.23134. We do not detect any other sources in the vicinity of Haumea, although we are sensitive to objects as faint as $0.11 \times$ the $J$-band (and $0.21 \times$ the $H$-band) flux of Hi'iaka. At the time of observation, the fainter, inner satellite Namaka was within $0.2^{\prime \prime}$ of Haumea (Ragozzine, private comm.), which explains why it is invisible in our data. Given its fractional brightness $(\sim 1 \%)$ with respect to Haumea, Namaka has negligible contribution to the near-infrared photometry presented here.

Haumea has a color index $J-H=-0.057 \pm 0.016 \mathrm{mag}$ (see Fig. 3 and Table 6). Our photometry of Hi'iaka relative to Haumea implies a color $J-H=-0.399 \pm 0.034$ mag for the former. This color index is unusually blue, but consistent with the observation that the $1.5 \mu \mathrm{m}$ band is deeper for Hi'iaka than for Haumea (Takato et al. 2006; Barkume et al. 2006). Table 6 lists the derived colors of Haumea and Hi'iaka. A sample of $J-H$ colors of $40 \mathrm{KBOs}$ and Centaurs found in the literature (Delsanti et al. 2006) shows a pronounced clustering around $J-H=0.4 \mathrm{mag}$ with a dispersion of $\sim 0.2$ mag. The two significant outliers are KBOs (19308) 1996 $\mathrm{TO}_{66}(J-H=-0.21 \pm 0.17 \mathrm{mag})$ and (24835) 1995 $\mathrm{SM}_{55}(J-H=-0.49 \pm 0.06 \mathrm{mag})$ which both possess near-infrared spectra consistent with water-ice absorption (Brown et al. 1999; Barkume et al. 2008).

The $J-H$ colors of Haumea and Hi'iaka are significantly $(>9 \sigma)$ different. Given the current best estimates for the mass and orbit of Hi'iaka, particles collisionally ejected (ejection velocities 10 to $200 \mathrm{~m} \mathrm{~s}^{-1}$ ) from its surface will likely reach Haumea only on hyperbolic orbits (Stern 2008). The escape speed from the surface of Hi'iaka (near $130 \mathrm{~m} \mathrm{~s}^{-1}$, assuming water-ice density) rivals the escape speed from the Haumea system at the orbit of Hi'iaka $\left(\sim 120 \mathrm{~m} \mathrm{~s}^{-1}\right)$, meaning that only hyperbolic mass exchange is possible. The same cannot be said about Namaka which is both smaller and deeper into the potential well of the satellite system. It is therefore more likely that Haumea is polluted by ejecta from Namaka than from Hi'iaka. Whether this means that the $J-H$ color of Namaka is closer to that of Haumea remains to be seen.

\section{SUMMARY}

From time-resolved, near-infared photometry of Kuiper belt object Haumea we find the following

- The near-infrared peak-to-peak photometric range is $\Delta m_{R}=0.30 \pm 0.02$ mag. The new data reveal slight visible/near-infrared color variations on Haumea, which are spatially correlated with a previously identified surface region, redder in $B-R$ and darker than the mean surface. We find that near this region Haumea displays an enhanced $H$-band reflectance accompanied by $B$-band absorption relative to elsewhere on the surface. Time-resolved spectra are needed to learn more about the physicochemical properties of this anomalous region.

- The rotationally medianed visible and nearinfrared colors of Haumea are $R-J=0.885 \pm 0.012$ mag and $J-H=-0.057 \pm 0.016$ mag. 

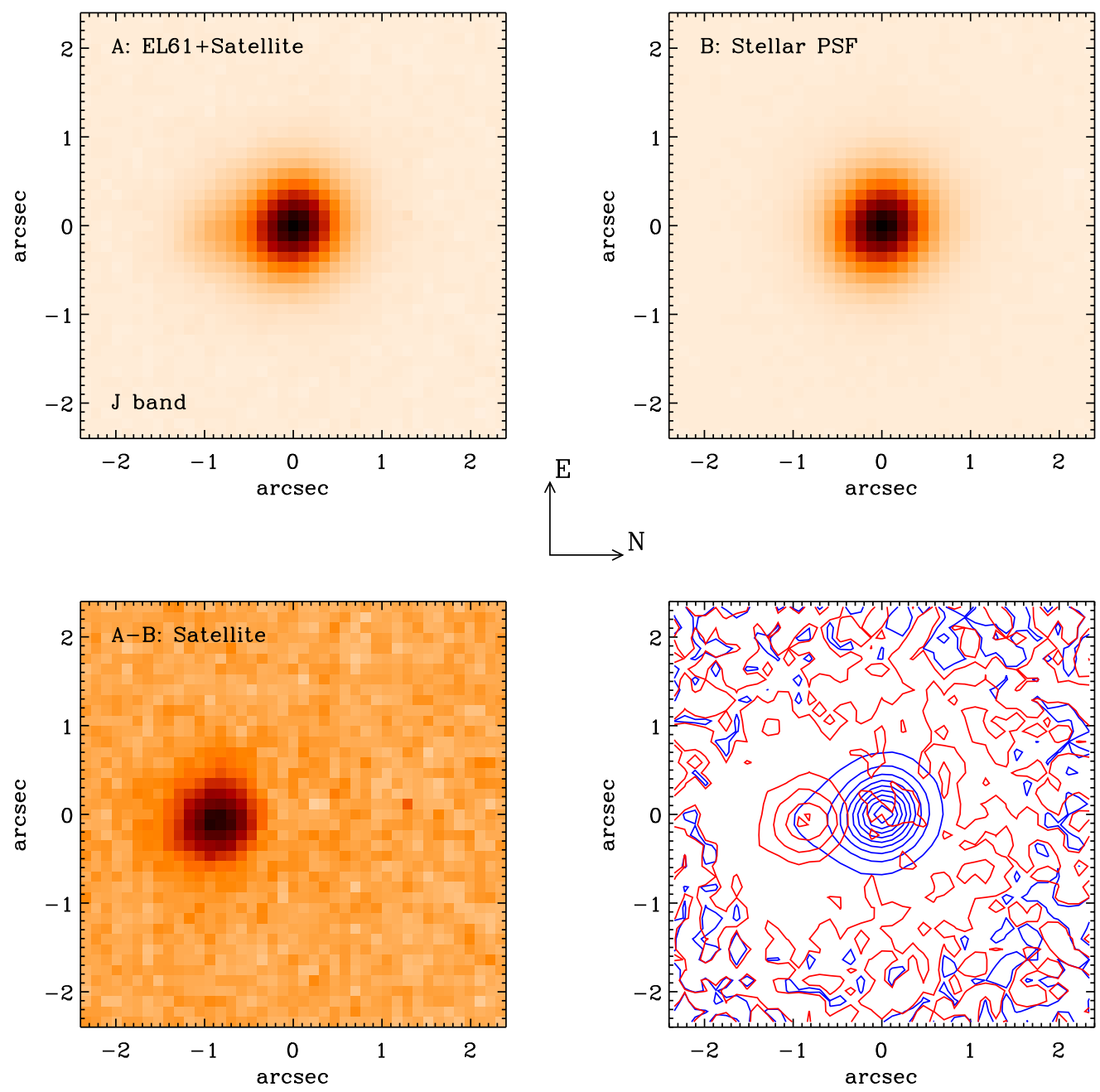

FIG. 9.- Top left: Stack of $\sim 70 \mathrm{~J}$-band frames of Haumea. The satellite Hi'iaka is discernible to the left of Haumea. Top right: Stellar PSF built from a stack of $\sim 70 \mathrm{~J}$-band frames of a star in the Haumea field. Bottom left: Image of Hi'iaka obtained by subtracting the top right image from the top left one. Bottom right: Contours of Haumea and Hi'iaka (blue) overplotted on contours of the satellite Hi'iaka alone (red) to show the relative position.

- We detect Hi'iaka, Haumea's brightest satellite, in both $J$ and $H$ and measure its color $J-H=$ $-0.399 \pm 0.034 \mathrm{mag}$. The $J-H$ color difference between Hi'iaka and Haumea is significant $(>9 \sigma)$. This suggests that either the transfer of surface ejecta between the two is negligible, or that their surface colors are not controlled by ejecta transfer. Ejecta transfer between Haumea and the inner satellite Namaka is neither ruled out nor substantiated by our data but is more likely given the configuration of the system.

- The slope of the $J$-band phase function in the range $0.55 \leq \alpha(\mathrm{deg}) \leq 1.14$ is $\beta_{J}=0.16 \pm 0.04 \mathrm{mag}$ $\mathrm{deg}^{-1}$. Combining this measurement with slopes obtained in three other visible wavelengths we find that the slope of Haumea's phase function varies monotonically with wavelength. The slope of the relation is $\Delta \beta / \Delta \lambda \sim 0.09 \mathrm{mag} \mathrm{deg}^{-1} \mu \mathrm{m}^{-1}$ and $\beta_{1 \mu \mathrm{m}} \sim 0.14 \mathrm{mag} \mathrm{deg}^{-1}$. This finding confirms previous inferences that coherent backscattering is the main cause of opposition brightening for Haumea.

\section{ACKNOWLEDGMENTS}

We appreciate insightful discussion and comments from David Jewitt and Jan Kleyna. We thank Will Grundy for sharing useful software routines, and Jon Swift for valuable discussion that contributed to this work. PL was supported by a grant to David Jewitt from the National Science Foundation.

\section{REFERENCES}

Bagnulo, S., Boehnhardt, H., Muinonen, K., Kolokolova, L., Belskaya, I., \& Barucci, M. A. 2006, A\&A, 450, 1239
Barkume, K. M., Brown, M. E., \& Schaller, E. L. 2006, ApJ, 640, L87

-. 2008, AJ, 135, 55 

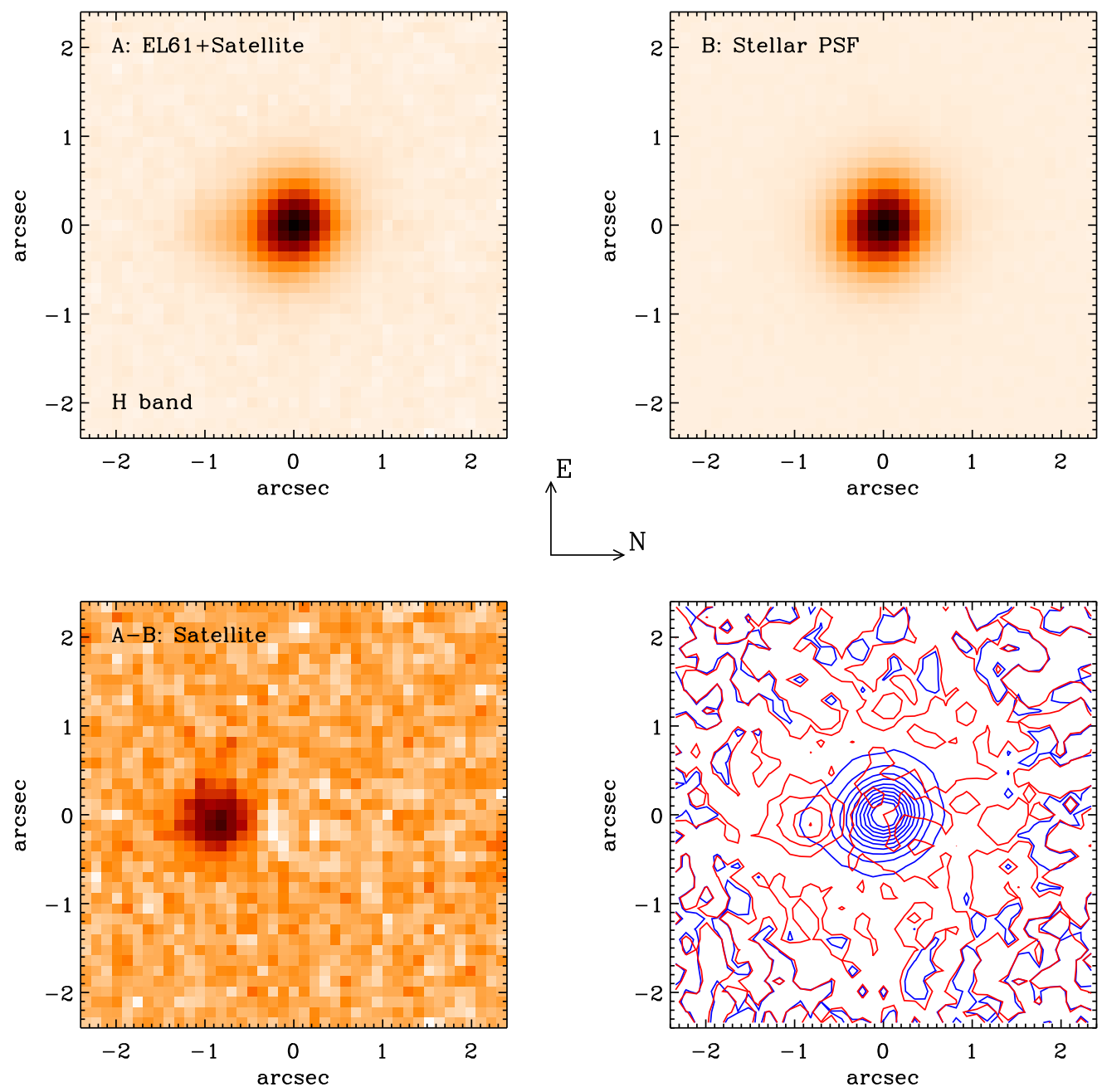

FIG. 10.- Same as Fig. 9 for $H$-band data.

Boehnhardt, H., Bagnulo, S., Muinonen, K., Barucci, M. A. Kolokolova, L., Dotto, E., \& Tozzi, G. P. 2004, A\&A, 415, L21 Brown, M. E., Barkume, K. M., Ragozzine, D., \& Schaller, E. L. 2007, Nature, 446, 294

Brown, M. E., Trujillo, C. A., \& Rabinowitz, D. L. 2005, ApJ, 635, L97

Brown, M. E., et al. 2006, ApJ, 639, L43

Brown, R. H., Cruikshank, D. P., \& Pendleton, Y. 1999, ApJ, 519, L101

Buratti, B. J., et al. 2003, Icarus, 162, 171

Cruikshank, D. P., Pilcher, C. B., \& Morrison, D. 1976, Science, 194, 835

Delsanti, A., Peixinho, N., Boehnhardt, H., Barucci, A., Merlin, F., Doressoundiram, A., \& Davies, J. K. 2006, AJ, 131, 1851

Feierberg, M. A., Lebofsky, L. A., \& Tholen, D. J. 1985, Icarus, 63, 183

French, R. G., Verbiscer, A., Salo, H., McGhee, C., \& Dones, L. 2007, PASP, 119, 623

Gaffey, M. J., \& McCord, T. B. 1978, Space Science Reviews, 21, 555

Grundy, W. M., \& Schmitt, B. 1998, J. Geophys. Res., 103, 25809

Hapke, B. 1993, Theory of reflectance and emittance spectroscopy (Topics in Remote Sensing, Cambridge, UK: Cambridge University Press, —c1993)

Hapke, B., Nelson, R., \& Smythe, W. 1998, Icarus, 133, 89

Hawarden, T. G., Leggett, S. K., Letawsky, M. B., Ballantyne, D. R., \& Casali, M. M. 2001, MNRAS, 325, 563
Helfenstein, P., et al. 1998, Icarus, 135, 41

Holmberg, J., Flynn, C., \& Portinari, L. 2006, MNRAS, 367, 449 Jewitt, D., Peixinho, N., \& Hsieh, H. H. 2007, AJ, 134, 2046

Karkoschka, E. 2001, Icarus, 151, 51

Lacerda, P., Jewitt, D., \& Peixinho, N. 2008, AJ, 135, 1749

Lacerda, P., \& Jewitt, D. C. 2007, AJ, 133, 1393

Licandro, J., Pinilla-Alonso, N., Pedani, M., Oliva, E., Tozzi, G. P., \& Grundy, W. M. 2006, A\&A, 445, L35

Malhotra, R. 1995, AJ, 110, 420

Merk, R., \& Prialnik, D. 2006, Icarus, 183, 283

Nelson, R. M., Hapke, B. W., Smythe, W. D., \& Horn, L. J. 1998, Icarus, 131, 223

Nelson, R. M., Hapke, B. W., Smythe, W. D., \& Spilker, L. J. 2000, Icarus, 147, 545

Rabinowitz, D. L., Barkume, K., Brown, M. E., Roe, H., Schwartz, M., Tourtellotte, S., \& Trujillo, C. 2006, ApJ, 639, 1238

Rabinowitz, D. L., Schaefer, B. E., \& Tourtellotte, S. W. 2007, AJ, 133, 26

Ragozzine, D., \& Brown, M. E. 2007, AJ, 134, 2160

Stern, S. A. 2008, ArXiv e-prints, 805

Takato, N., Terada, H., \& Tae-Soo, P. 2006, Oral communication Tegler, S. C., Grundy, W. M., Romanishin, W., Consolmagno, G. J., Mogren, K., \& Vilas, F. 2007, AJ, 133, 526

Tholen, D. J., \& Barucci, M. A. 1989, in Asteroids II, ed. R. P. Binzel, T. Gehrels, \& M. S. Matthews, 298-315 
Tokoku, C., et al. 2003, in Instrument Design and Performance for Optical/Infrared Ground-based Telescopes., ed. M. Iye \& A. F. M. Moorwood, Vol. 4841, 1625-1633

Trujillo, C. A., Brown, M. E., Barkume, K. M., Schaller, E. L., \& Rabinowitz, D. L. 2007, ApJ, 655, 1172 